\title{
HOW DO WE PERCEIVE EMOTION FROM A 3-D VIRTUAL TALKING HEAD: EVIDENCE FROM FUNCTIONAL NEAR-INFRARED SPECTROSCOPY
}

\author{
Jianying Wang ${ }^{1}$, Jiangtao Chen ${ }^{1}$, Nan $\mathrm{Yan}^{1}$, Lan Wang ${ }^{1}$ and Lawrence $\mathrm{Ng}^{2}$ \\ ${ }^{I}$ CAS Key Laboratory of Human-Machine Intelligence-Synergy Systems, Shenzhen Institutes of \\ Advanced Technology, Chinese Academy of Sciences \\ 1068 Xueyuan Boulevard, University Town of Shenzhen, Xili Nanshan, 518055, Shenzhen, China \\ ${ }^{2}$ Speech Science Laboratory, University of Hong Kong, Hong Kong, China
}

\begin{abstract}
Emotional interaction plays an important role in human-computer interaction. Although more and more virtual heads are endowed with a series of facial expressions under the conversational scenarios, the similarities and differences in neural mechanism underlying emotional perception between virtual head and human are still largely unknown. In the present study, we investigated the activation of emotional perception activities in the dorsolateral prefrontal cortex (DLPFC) region associated with when humans when beings perceiving emotions from 3-D virtual talking heads by using functional near-infrared spectroscopy (fNIRS). Three dynamic emotional stimuli were consisting of 3-D virtual talking head and human face with mute mode or voice presented by twenty participants. The behavioral results showed that participants had lower accuracy watching mute 3-D virtual talking head videos (3DMute) than watching human face videos with voice (HFMute) especially for in anger and happiness stimuli except for neutral emotion. The fNIRS results observed that there was no difference in DLPFC activity area for the observation of 3-D virtual talking head and human face. However, it was found that a stronger DLPFC region had stronger activation was observed for 3-D virtual talking head videos with voice (3DVoice) than 3DMute. In addition, the a stronger activation in DLPFC activation region for affectively angry emotion was videos observed in females was demonstrated, but yet there was no gender difference was found in gender during watching both types for happiness videos. The present results work provided preliminary evidence in physiology mechanism in human-computer interaction.
\end{abstract}

\section{KEYWORDS}

Human-Computer Interaction, Emotional Perception, Function Near-Infrared Spectroscopy, 3-D Virtual Talking Head, Dorsolateral Prefrontal Cortex

\section{INTRODUCTION}

Emotion perception yields a group of cognitive, physiological and motor changes, based on which an individual could make conscious or unconscious decision (Etkin et al., 2015). Emotion interaction plays an important role in human-computer interaction. Nowadays more and more virtual heads are endowed with a repertoire of facial expressions in conversation scenarios, however, how do humans perceive emotion of a virtual head? Is such emotion perception the same as perceiving emotion from a human face?

Magalie et al. (2015) explored emotive facial expressions using morphological and dynamic characteristics and measured the effectiveness of user's perception of the emotive facial expression during human-machine interaction. Kätsyri et al. (2008) compared emotion recognition using synthetic and natural human facial expressions, they and found a lower accuracy in recognizing synthetic expressions than natural expressions. Such discrepancy was believed to originate from the low intensity of the expressions and the imperfect emotion model of the synthetic expressions.

Despite the studies of behavioral responses associated with emotion perception, the corresponding cortical responses are still largely unknown. Brain functional imaging is a noninvasive method that can be used to record brain activity during emotion processing (Etkin et al., 2011, Glotzbach et al., 2011), and thus provide an objective account of emotion perception by visualizing the neural activity a reality. In comparison to 
behavioral responses, emotion-related neural activity provides a more solid ground for the study of emotion perception (Ekman et al., 1994). Recently, Wabnegger et al. (2015) applied functional magnetic resonance imaging (fMRI) to study facial emotion recognition in patients with Parkinson. The clinical group showed a generally stronger activation in emotion-related neural activity regions compared to the healthy controls. Balconi et al. (2016) applied functional near-infrared spectroscopy (fNIRS) and electroencephalography (EEG) to explore the cortical lateralization effect in dorsolateral prefrontal cortex (DLPFC) region of the brain with emotional visual and auditory stimulation. They confirmed that the important role of the DLPFC in processing emotional interactions also worked in different species.

fNIRS is a novel, non-invasive brain functional imaging technique with a good temporal resolution (Robert et al., 2016). The technique is mainly used to detect the hemodynamic activity of the cerebral cortex. Despite the limited detection of depth of cerebral cortex associated with fNIRS (Marco et al., 2012), most researches focused on the prefrontal cortex (PFC) which has been confirmed to be involved in emotion processing (Nishitani et al., 2013). Miranda et al. (2015) used images of happy, fearful and angry female facial expressions as stimuli to explore the differences emotion response in infant with focus on the prefrontal cortex when watching the same facial expression. They observed that the activities of PFC were negatively correlated with temperamental factors when the infants watched a happy facial expression. Wrase et al. (2003) studied the difference in PFC activity between genders when reacting to positive and negative emotions, and found a stronger activity in PFC region for happiness videos in men, and stronger activation in PFC region for affectively anger videos in women. The PFC region was demonstrated to be related with emotion.

However, it is not known if similar PFC involvement exists for perception of emotion of a virtual head. Recently, by using fNIRS, Aranyi et al. (2016) observed participants' response in the DLPFC region when perceiving the virtual agent showing different facial expressions. The participants' response showed activation in DLPFC, consistent with what they expected. These findings confirmed the impact of virtual agent on people's emotional perception. Yet, emotion perception of virtual agent with a human face was not included. It is still not known if similar findings could be found for perceiving an emotion from a virtual agent through using a human face. In fact, such emotion perception has been of interest to many researchers nowadays, and some brain regions have been found to be relevant in emotional processing. Matsubara et al. (2014) reported that the DLPFC region played an essential role in the top-down cognitive-emotion control of affect-generating structures. The neural processing of emotion regulation was associated with the model of emotion (Gross, 1998), according to which the ventrolateral prefrontal cortex (VLPFC) region estimates the affection and signals the need for regulatory processing via anterior middle cingulate cortex (aMCC) or its direct anatomical connection to DLPFC. The DLPFC processes the regulation and provides a feed forward signal to angular gyrus, supplementary motor area (SMA), superior temporal gyrus (STG), amygdala and basal ganglia, which in turn executes the emotion regulation (Gross \& Thompson, 2007; Kohn et al., 2014).

In our previous work, a 3-D virtual talking head driven by real physical data with human emotions has been developed, and eye tracking experiments found no significant difference in fixation duration when watching 3-D virtual talking head videos and human face videos (Ni et al., 2018). As an extension of such, the present study investigated brain activation when subjects perceived different emotions from a 3-D virtual talking head by using fNIRS technology. Dynamic emotion stimuli (anger, happiness and neutral) conveyed by: (1) mute 3-D virtual talking head videos (3DMute), (2) 3-D virtual talking head videos with voice (3DVoice), (3) mute human face videos (HFMute), and (4) human face videos with voice (HFVoice) were used. The present study set out to confirm that DLPFC, as part of PFC, is an area strongly associated with emotions. In addition, accuracy in recognizing emotions in the above four scenarios was also analyzed. Based on the above discussion, three hypotheses were set up. Firstly, there was no significant difference when people were watching 3-D virtual talking head and human face. Secondly, the previous research by Sharon et al. (2013) found stronger activation in infants when watching the smile of their mother than others. It was also hypothesized that easier distinction of stimuli with improved activation of DLPFC could be found when viewing videos with voice than mute videos. Thirdly, for happiness emotion, it was hypothesized that stronger activation in DLPFC region for affectively when women watched videos of anger emotion.

The rest of this paper is organized as follows: Section 2 and 3 presents the method and data analysis respectively. The results and discussion are presented in Section 4 and 5. The final Section draws the conclusion and the future work. 


\section{METHOD}

\subsection{Participants}

A group of 20 healthy Chinese college students were recruited in this study including 10 males (age: $\mathrm{M}=24.60$ years, $\mathrm{SD}=1.955$ years) and 10 females (age: $\mathrm{M}=23.70$ years, $\mathrm{SD}=1.059$ years). All participants were right handed and had either normal or corrected vision. All participants were informed about the purpose and the safety of the experiment, and a written informed consent was obtained. The study was approved by the Institutional Review Board of Shenzhen Institutes of Advanced Technology Chinese Academy of Sciences.

\subsection{Experimental Design}

For stimulus-induced activation, two experiments from the stimuli material covering two kinds of videos with two different modes, which were 3DMute, 3DVoice, HFMute and HFVoice were selected. A total of 60 videos with different showing time ranging from $2.5 \mathrm{~s}$ to $10.7 \mathrm{~s}$ were obtained. They included three emotions (anger, happiness and neutral). Among the videos, each emotion was shown in five videos with different speech content, and the speech content of 3-D virtual talking head videos corresponded to human face videos. The experiment used the event-related design and stimuli were presented randomly. Six conditions (2 renderings: 3 -D virtual talking head video and human face video x 3 emotions: anger, happiness and neutral) were set. The block consisted of 60 trails and each trial consisted four epochs including fixation time, viewing time, choose time and blank time. The fixation time was set at $1 \mathrm{~s}$ and the view time depended on the videos. Participants had the time to choose a right emotion of the presented stimuli. Blank time was set at 20s. To investigate the activation of emotional perception in DLPFC region when human beings perceive emotions from 3-D virtual talking head by fNIRS, the behavioral data and fNIRS data were recorded.

\subsection{Instrumentation}

To obtain fNIRS data, the NIRScout System (NIRx Medical Technologies, LLC) was used to simultaneously measure changes in concentration of oxygenated hemoglobin and deoxygenated hemoglobin. During the experiment, a plate was attached to the cap made of sturdy fabric to the participants. In each patch, eight detectors and seven light sources were embedded, resulting in 20 measurement channels $(\mathrm{CH})$, as shown in Figure 1. The source detector distance was $3 \mathrm{~cm}$ and a sampling frequency of $7.81250 \mathrm{~Hz}$ was used.

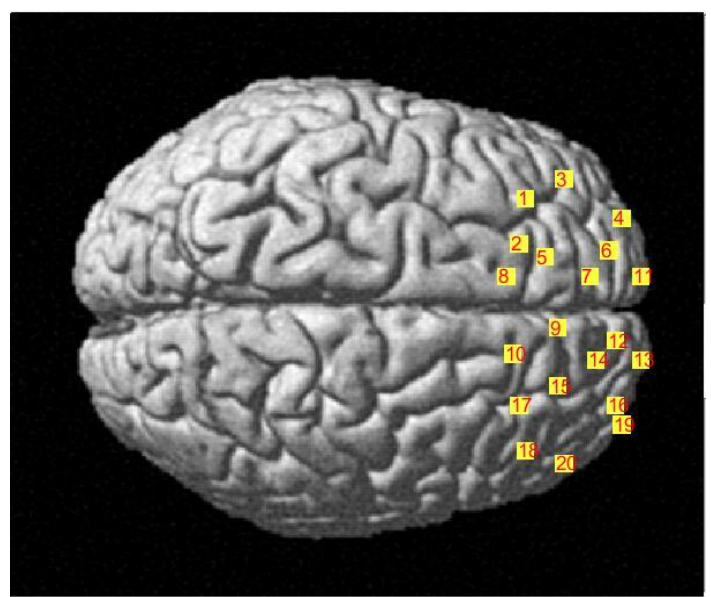

Figure 1. The configuration of channels 


\subsection{Procedure}

During the experiment, the participant was seated in the front of a 22 -in screen at the distance of $60 \mathrm{~cm}$ from the screen displaying the visual stimuli inside a quiet, dimly lit room. The video stimuli were displayed using E-Prime 2.0 software (Psychology Software Tools, Inc.) with which behavior data were recorded. The participants were first instructed about their task with instructions displayed on the computer screen. The sequence of experiment is shown in Figure 2 and the key frame picture of the animation sequence of happiness and anger emotion perception is shown in Figure 3. The participants were then instructed to carry out the first experiment during which they watched two types of mute videos presented at a random order. After each video stimulus was presented, the participant recognized the emotion and selected their choice by pressing the serial response box with three keys corresponding to three different emotions. The first experiment completed when 60 trials were presented on the computer screen. A brief period of time-out was provided to the participants upon completion of the first experiment. The procedure of the second experiment was similar to the first, except that stimuli with videos and voice were used.

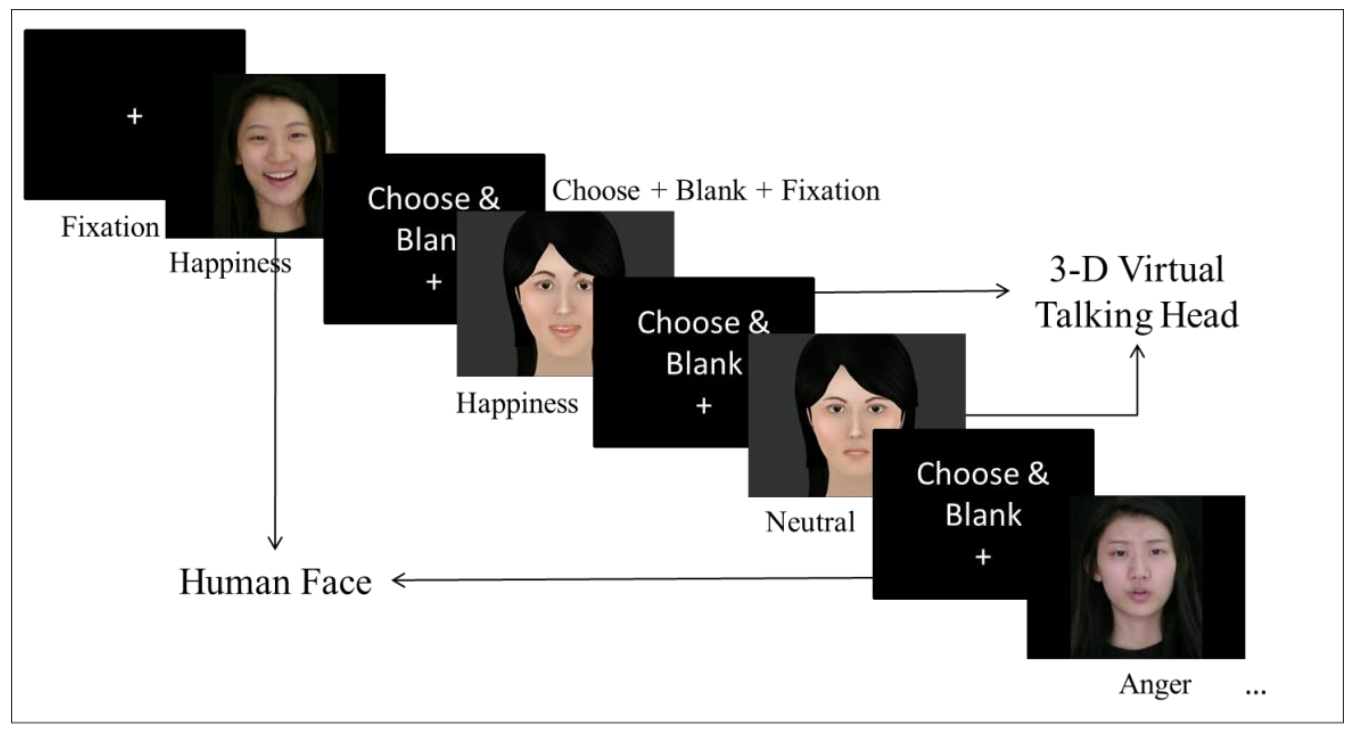

Figure 2. The sample of experiment

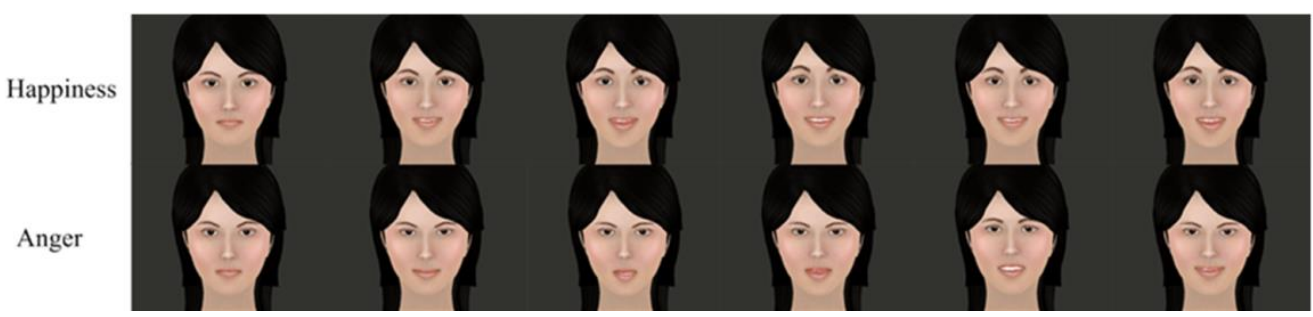

Figure 3. The key frame picture of the animation sequence of happiness and anger

\section{DATA ANALYSIS}

Before generating hemodynamic states data of each participant, the raw data was preprocessed by using nirsLAB (Xu et al., 2014). Data preprocessing consisted of: (1) interpolation of detector saturation, (2) checking data quality, (3) removal of discontinuities, (4) removal of spike artifacts, and (5) application of frequency filter. The time series of hemodynamic states including oxygenated hemoglobin was computed from the filtered data produced by the preprocessing utility based on the Beer-Lambert Law. Finally, NIRS-SPM 
(Ye et al., 2009) was applied to generate a General Linear Model (GLM) matrix and functional data can be modeled using the equation:

$$
\mathbf{Y}=\mathbf{X} * \boldsymbol{\beta}+\varepsilon
$$

where $\mathbf{Y}$ is the matrix of hemodynamic data, $\mathbf{X}$ constitutes the design matrix containing the respective modeled effects, $\beta$ is the parameter matrix comprising the beta weights to be estimated and the residual term $\varepsilon$. More precisely, the design matrix $\mathbf{X}$ of modeled effects comprises the predicted hemodynamic response function for each experimental condition over time, with stimulus onset as starting point. Hence, the oxyhaemoglobin concentration response of each condition of each participant will be estimated.

To estimate parameter $\hat{\boldsymbol{\beta}}$, some parameters was set: basic function was 'hrf', Filtering was discrete cosine transform (DCT) with cutoff frequency $1 / 128 \mathrm{~s}=0.008 \mathrm{~Hz}$ to ensure that the DCT band does not overlap with any event related frequencies. Gaussian smoothing with FWHM 4s to process ship type related noise.

\section{RESULT}

\subsection{The Behavioral Data}

Recognition accuracy associated with perceiving different emotions and different types of rendering by males and females are shown in Figures 4 and 5.

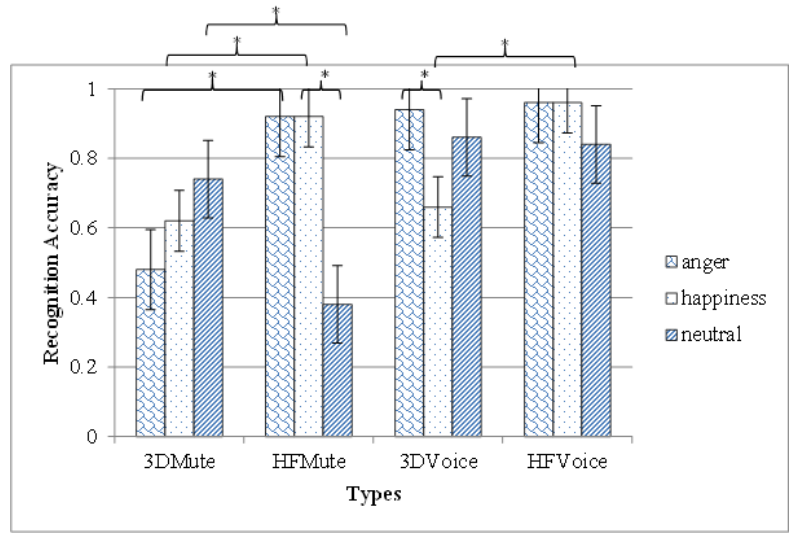

Figure 4. Recognition accuracy of male group

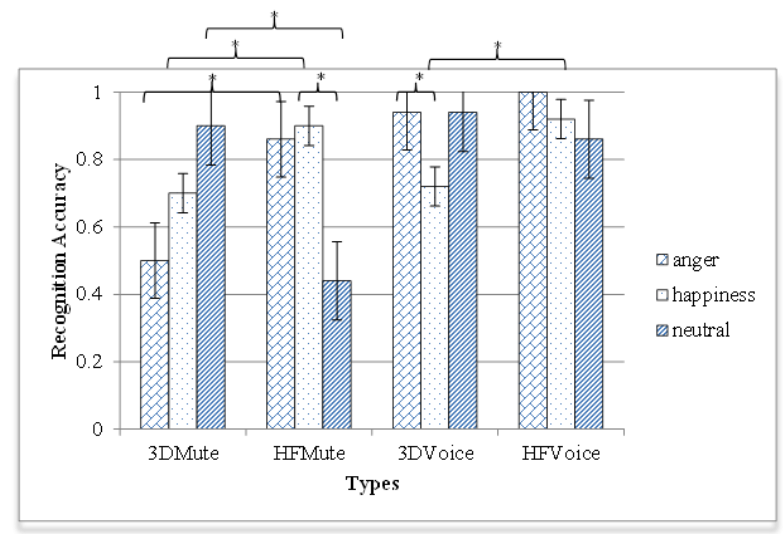

Figure 5. Recognition accuracy of female group 
To determine whether the type of rendering (3-D virtual talking head videos and human face videos), mode (videos with voice and the mute videos) and gender (male and female) differed on different emotions, multiple-way analysis of variance (ANOVA) (Emotion $*$ Type $*$ Mode $*$ Gender) was conducted on recognition accuracy of dynamic expressions.

There was main effect in mode $(\mathrm{F}(1,216)=59.108, \mathrm{p}<0.001)$, type $(\mathrm{F}(1,216)=10.857, \mathrm{p}=0.001)$ and emotion $(\mathrm{F}(2,216)=3.788, \mathrm{p}=0.024)$. But there was no significant difference between the recognition accuracy of male and female.

There was no significant difference between the recognition accuracy of the videos with voice and the mute videos, and watching the videos with voice had higher accuracy than the mute videos $(\mathrm{F}(1,216)=59.108$, $\mathrm{p}<0.001)$. Besides, there was interaction effect between mode and emotion $(\mathrm{F}(2,216)=10.423, \mathrm{p}<0.001)$. When watching the mute videos, watching happiness had higher accuracy than neutral $(\mathrm{F}(2,216)=8.208$, $\mathrm{p}<0.001)$. When watching the videos with voice, watching anger had higher accuracy than happiness $(\mathrm{F}(2,216)=6.003, \mathrm{p}=0.002)$. There was interaction among type and mode and emotion $(\mathrm{F}(2,216)=18.320$, $\mathrm{p}<0.001)$. When the emotion was anger, watching 3DMute had lower accuracy than HFMute $(\mathrm{F}(1,216)=45.236, \mathrm{p}<0.001)$. And for the emotion was happiness, watching 3DMute had lower accuracy than HFMute $(\mathrm{F}(1,216)=17.670, \mathrm{p}<0.001)$. However, when the emotion was neutral, watching 3DMute had higher accuracy than HFMute $(\mathrm{F}(1,216)=47.526, \mathrm{p}<0.001)$. Watching 3DVoice had lower accuracy than HFVoice $(\mathrm{F}(1,216)=17.670, \mathrm{p}<0.001)$ when the emotion was happiness.

\section{2 fNIRS Data}

Oxyhaemoglobin concentration response was recorded by fNIRS, and the activated region of group can be seen directly as shown in Figure 6.

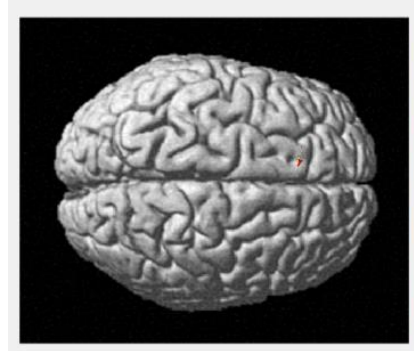

(a)

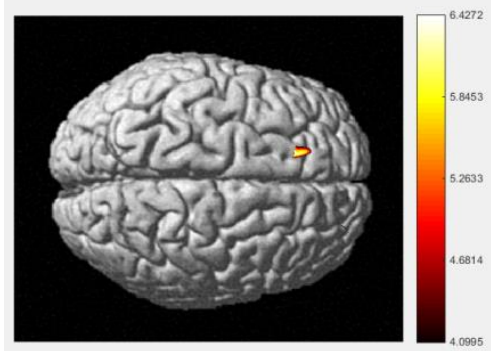

(b)

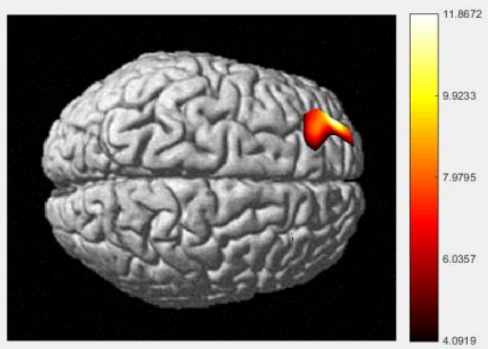

(c)

Figure 6 . The group activation patterns ( $p<0.05$, uncorrected) from oxyhaemoglobin concentration response with contrast watching 3 -D talking head videos and human face videos with the emotion is anger (a), happiness

(b) and neutral (c)

To compare the difference between human face videos and 3-D talking head videos, oxyhaemoglobin concentration response was analyzed with the one way ANOVA in type. It was found that there was no significant difference between watching human face videos and 3-D talking head videos at all channels. Oxyhaemoglobin concentration response for watching human face videos was analyzed with multi-way ANOVA (Emotion*Mode*Gender). There was main effect in gender at $\mathrm{CH} 3(\mathrm{~F}(1,34)=5.089, \mathrm{p}=0.039)$ and $\mathrm{CH} 6(\mathrm{~F}(1,34)=5.358, \mathrm{p}=0.035)$. Also emotion had main effect at $\mathrm{CH} 3(\mathrm{~F}(2,34)=5.234, \mathrm{p}=0.019)$ and $\mathrm{CH} 6(\mathrm{~F}(2,34)=5.437, \mathrm{p}=0.017)$. Further, there was interaction effect between gender and emotion at $\mathrm{CH} 13(\mathrm{~F}(2,34)=4.109, \mathrm{p}=0.038)$ and $\mathrm{CH} 18(\mathrm{~F}(2,34)=4.249, \mathrm{p}=0.035)$. When the female group watched the expression videos, the activation of watching anger videos was higher than watching neutral videos at $\mathrm{CH} 18$. Besides, when the participants watched the same anger expression video, the activation of the prefrontal area of the male group was lower than female group. At the same time, there was interaction effect in mode and emotion at $\mathrm{CH} 6(\mathrm{~F}(2,34)=4.276, \mathrm{p}=0.0034)$ and $\mathrm{CH} 13(\mathrm{~F}(2,34)=6.647, \mathrm{p}=0.009)$.

Oxyhaemoglobin concentration response for 3-D virtual talking head was analyzed using multiple-way ANOVA (Emotion* Mode*Gender). There was main effect in mode at $\mathrm{CH} 14(\mathrm{~F}(1,34)=5.044, \mathrm{p}=0.031)$, $\mathrm{CH} 17(\mathrm{~F}(1,34)=9.259, \mathrm{p}=0.004)$ and $\mathrm{CH} 20(\mathrm{~F}(1,34)=5.246, \mathrm{p}=0.028)$. And the activation of watching the 3DMute was lower than 3DVoice. When the female group watched the expression videos at $\mathrm{CH} 17$, the activation of watching the 3DMute was lower than 3DVoice. 


\section{DISCUSSION}

The present study investigated emotion recognition and activation of emotion perception in the DLPFC region when perceiving different types of emotions from 3-D virtual talking head by fNIRS under twelve conditions - three emotions (angry, happiness and neutral) in mute 3-D virtual talking head videos (3DMute), 3-D virtual talking head videos with voice (3DVoice), human face videos with voice (HFMute) and mute human face videos (HFV oice). The behavioral and fNIRS data were compared to study the similarities and differences in neural mechanism underlying emotional perception between virtual head and human.

From the behavioral data, accuracy of recognizing anger and happiness emotions when watching 3-D talking head videos had lower accuracy than videos of human face. This is in line with the previous findings (Kätsyri et al., 2008). But, for neutral emotion, watching 3-D talking head videos had higher accuracy than human face videos.

Although watching 3-D talking head videos had lower accuracy than human face videos, there was no significant difference of DLPFC activation between human face videos and 3-D talking head videos. This finding reveals a similar cortical mechanism for emotion perception of both 3-D virtual talking head videos and human face videos. This is partly in line with the previous research by $\mathrm{Ni}$ et al. (2018), in which no significant difference in fixation duration when watching both types of videos. The present results also shed light in the understanding of the physiology for emotive interaction.

In addition, it is of interest to find that the activation in the DLPFC region when watching 3DVoice was stronger than 3DMute. Results were consistent with previous research (Sharon et al., 2013) with the key finding that infants had stronger activation when watching the smile of their mother than the others. The results indicated that stimuli of easier distinction may be improved to activate the DLPFC region.

Wrase et al. (2003) found that the stronger activity in PFC region for happiness videos in men, and stronger activation in the PFC region for affectively anger videos in women. The present data also showed a stronger activation in the DLPFC region for affectively anger videos perceived by women, but no significant difference in gender during watching both stimuli for happiness videos.

The present is a preliminary study of emotion perception by humans based on different types of renderings. Some limitations are observed. Firstly, the emotional recognition accuracy of 3-D virtual talking head videos was slightly lower than human face videos, the emotion expressed by 3-D virtual talking head should to be improved. Secondly, emotion intensity is important to emotional perception and the influence of emotion intensity of 3-D virtual talking head will be explored in future work.

\section{CONCLUSION}

Although watching 3-D talking head videos had lower accuracy than human face videos, there was a lack of significant difference of the activation in DLPFC region between human face videos and 3-D talking head videos. The DLPFC region saw a stronger activation when watching 3DVoice than 3DMute. In addition, the stronger activation in DLPFC region for affectively anger videos in female, but there was no difference between genders when watching videos of happiness emotion. The present work provided preliminary evidence in the physiology mechanism in human-computer interaction.

In the future, oxyhaemoglobin concentration response in DLPFC will be observed when individuals with autism spectrum disorders are watching 3-D talking head by fNIRS. As most researches explored the emotion perception of autism spectrum disorders to robots or virtual head by eye tracker (Bekele et al., 2013), these researches may be a new rehabilitation approach for autism spectrum disorders and improve their life.

\section{ACKNOWLEDGEMENT}

This study was jointly supported by a grant from National Natural Science Foundation of China (NSFC 61771461 and U1736202), Shenzhen Speech Rehabilitation Technology Laboratory and Health and Health Services Research Fund (HHSRF), Shenzhen Fundamental Research Program JCYJ20160429184226930, KQJSCX20170731163308665, JCYJ20170413161611534 and JCYJ20150330102401089. 


\section{REFERENCES}

Aranyi G, Pecune F, Charles F, et al., 2016. Affective interaction with a virtual character through an fnirs brain-computer interface, Frontiers in Computational Neuroscience, Vol. 10, No. 70.

Balconi, Michela, and Maria Elide Vanutelli, 2016. Hemodynamic (fNIRS) and EEG (N200) correlates of emotional inter-species interactions modulated by visual and auditory stimulation. Scientific reports, Vol .6, pp. 23083.

Bekele E, Zheng Z, Swanson A, et al., 2013. Understanding how adolescents with autism respond to facial expressions in virtual reality environments, IEEE transactions on visualization and computer graphics, Vol. 19, No. 4, pp. 711-720.

Ekman, Paul Ed, and Richard J. Davidson, 1994. The Nature of Emotion: Fundamental Questions, London, U.K.: Oxford Univ. Press.

Etkin, Amit, Christian Büchel, and James J. Gross, 2015. The neural bases of emotion regulation. Nature reviews neuroscience, Vol.16, No.11, pp. 693.

Etkin, Amit, Tobias Egner, and Raffael Kalisch, 2011. Emotional processing in anterior cingulate and medial prefrontal cortex, Trends Cognit. Sci., Vol. 15, No. 2, pp. 85-93.

Glotzbach, Evelyn, et al., 2011. Prefrontal brain activation during emotional processing: A functional near infrared spectroscopy study (fNIRS), The open neuroimaging journal, Vol. 5, pp. 33-39.

Gross, James J., 1998, The emerging field of emotion regulation: An integrative review. Review of general psychology, Vol.2, No.3, pp. 271-299.

Gross, James J., and Ross A.Thompson, 2007. Emotion regulation: Conceptual foundations. Handbook of Emotion Regulation, Vol.3, pp. 3-4.

$\mathrm{Hu} \mathrm{Ni}$, JianyingWang, LanWang, and Nan Yan, 2018. Track your emotional perception of 3-d virtual talking head in human-computer interaction, 2018 IEEE International Conference on Cyborg and Bionic Systems (CBS), pp. 298-303.

Jari Kätsyri and Mikko Sams, 2008. The effect of dynamics on identifying basic emotions from synthetic and natural faces, International Journal of Human-Computer Studies, Vol. 66, No. 4, pp. 233-242.

Kohn, Nils, et al., 2014. Neural network of cognitive emotion regulation—an ALE meta-analysis and MACM analysis. Neuroimage, Vol.87, pp.345-355.

Magalie Ochs, Radoslaw Niewiadomski, and Catherine Pelachaud, 2015. 18 Facial Expressions of Emotions for Virtual Characters. The Oxford Handbook of Affective Computing. Oxford Library of Psychology, Vol. 261.

Marco Ferrari and Valentina Quaresima, 2012. A brief review on the history of human functional near-infrared spectroscopy (fnirs) development and fields of application, Neuroimage, Vol. 63, No. 2, pp. 921-935.

Masataka, Nobuo, Leonid Perlovsky, and Kazuo Hiraki. 2015. Near-infrared spectroscopy (NIRS) in functional research of prefrontal cortex, Frontiers in human neuroscience, Vol.9, pp: 274.

Matsubara, Toshio, et al., 2014. Prefrontal activation in response to emotional words in patients with bipolar disorder and major depressive disorder, NeuroImage, Vol.85. pp:489-497.

Miranda M Ravicz, Katherine L Perdue, Alissa Westerlund, Ross E Vanderwert, and Charles A Nelson. 2015. Infants' neural responses to facial emotion in the prefrontal cortex are correlated with temperament: A functional near-infrared spectroscopy study, Frontiers in Psychology, Vol. 6, No. 922, pp. 922.

Nishitani, Shota, and Kazuyuki Shinohara, 2013. Nirs as a tool for assaying emotional function in the prefrontal cortex, Frontiers in Human Neuroscience, Vol. 7, No. 7, pp. 770.

Robert C. A. Bendall, Peter Eachus, and Catherine Thompson, 2016. A brief review of research using nearinfrared spectroscopy to measure activation of the prefrontal cortex during emotional processing: The importance of experimental design, Frontiers in Human Neuroscience, Vol. 10, No. 529, pp. 1-7.

Sharon Elizabeth Fox, Jennifer Wagner, Christine L Shrock, Helen Tager Flusberg, and Charles A Nelson, 2013. Neural processing of facial identity and emotion in infants at high-risk for autism spectrum disorders, Frontiers in human neuroscience, Vol. 7, pp. 89.

Wabnegger, Albert, Rottraut Ille, Petra Schwingenschuh, Petra Katschnig-Winter, Mariella Kögl-Wallner, Karoline Wenzel and Anne Schienle, 2015. Facial emotion recognition in Parkinson's disease: an fMRI investigation. PLoS One, Vol.10, No.8, pp. e0136110.

Wrase J, Klein S, Gruesser S M, et al., 2003. Gender differences in the processing of standardized emotional visual stimuli in humans: a functional magnetic resonance imaging study, Neuroscience Letters, Vol. 348, No. 1, pp. 41-45.

Xu, Yong, Harry L. Graber, and Randall L. Barbour, 2014. nirslab: a computing environment for fnirs neuroimaging data analysis, in Biomedical Optics. Optical Society of America, pp. BM3A-1.

Ye, Jong Chul, et al., 2009. Nirs-spm: statistical parametric mapping for near-infrared spectroscopy, Neuroimage, Vol. 44, No. 2, pp. 428-447. 